\title{
Numerical Simulation of Bending Stiffness Analysis for Spring Linkage Applied to In-Pipe Robot
}

\author{
Jinwei Qiao $(\mathbb{D})$ and Na Liu $(\mathbb{C}$ \\ School of Mechanical \& Automotive Engineering, Qilu University of Technology (Shandong Academy of Sciences), \\ Jinan 250353, China \\ Correspondence should be addressed to Jinwei Qiao; joe613722@126.com
}

Received 20 July 2020; Accepted 7 August 2020; Published 27 August 2020

Academic Editor: Yumin Cheng

Copyright (c) 2020 Jinwei Qiao and Na Liu. This is an open access article distributed under the Creative Commons Attribution License, which permits unrestricted use, distribution, and reproduction in any medium, provided the original work is properly cited.

\begin{abstract}
Spring linkage can be applied to in-pipe robots for connecting different modules together and can make it pass through elbows more easily. However, its stiffness cannot be set to be too hard or too soft. This paper tries to make a balance between the compressive stiffness and the bending stiffness of the spring. After a brief introduction to the construction mechanism and some assumptions, the mathematical representation of the spring bending stiffness was deduced based on the Kirchhoff theory which describes the spatial curve with displacement rather than time. Then, some simulations aiming at verifying the correctness of the deduced bending stiffness expression were carried out. Finally, the relationship between the two rigidities was found out, which helps to find a way to decrease the bending stiffness of spring while keeping its compressive stiffness strong enough.
\end{abstract}

\section{Introduction}

Many in-pipe robots have been widely applied to pipeline surveillance, detection, and maintenance [1-4]. In most cases, the in-pipe robot with only one module is always not sufficient enough to carry heavy payload or to pass through bent parts of a pipeline $[5,6]$. The linkage for connecting modules is incredibly necessary [7]. However, few concerns have been arisen. Reported linkages can be divided into two classes, the active and the passive $[8,9]$. As the name suggests the active one can change direction actively [10-12]. It has steering mechanism that usually consists of motor, transmission system, and the executor. For example, in [13], the Explorer Robot adopts motor actuated gear transmission box as its linkage to transmit not only force and motion but also torque, developed by the Robotics Institute at Carnegie Mellon University. Similar steering mechanisms appeared in [14-16]. The steering mechanism adds the weight of the robot body and is always quite complicated, too large to be used in closed pipeline. In view of this point, the passive one is better [17]. As the typical one, the universal joint has obtained the widest applications
[18] such as the robots described in $[19,20]$. It is found that the use of traditional joints often leads to some inconvenience, such as the enlarged volume, raised weight, and blocked transportation vessel among modules. In order to get through the blocked transformation, a specially designed universal joint was processed in [21]. It is quite inconvenient.

One alternative is the spring that was firstly introduced in [22]. With the addition of a ball in the updated version, it obtained the ability to pass though elbows. However, there would be pressure acting directly on the pipe wall, caused by bending connecting spring, as the in-pipe robot tries to pass through elbows. The pressure then turns to be resistant friction for moving robot. Therefore, the connecting spring cannot be designed to be too soft, which means large bending stiffness may push the two ends of spring against the pipeline hardly and create horrible friction [23, 24]. At the same time, the compressive stiffness of spring cannot be too small [25]. Effective transmitting of force and motion relies on strong compressive stiffness; otherwise, the transmitted force will be decreased and the transmitted motion will be delayed. It needs a balance. 
The numerical simulation is the main researching method for bending stiffness of spring linkage [26, 27]. There are many numerical methods in recent years, which mainly include finite element method (FEM) [28, 29], boundary element method (BEM) [30, 31], and meshless method [32-37]. The FEM is the most widely used numerical simulation method, but in the simulation of nonlinear problems, the meshless method and the BEM have more advantages than the finite element method $[38,39]$. In this paper, the FEM is used to simulate bending stiffness of spring linkage.

The rest of this paper is mainly organized as follows. Firstly, it introduces the basic concept of the linkage mechanism and explains its advantages. Then, the expression for spring stiffness is deduced, followed by some simulations and discussion. The conclusion obtained from this work is given at last.

\section{Mechanism Description}

As shown in Figure 1, two separate modules of in-pipe robots with end screw threads are connected using a compressible round coil spring. The screw pitches $l_{1}$ of the two modules share the same size, larger than that of the spring, termed as $l_{2}$, while the bottom diameter of the screw thread and the inner diameter of the spring share the same size. Therefore, the spring pitch could be elongated when engaged with screw thread, so that the tensile force from lengthened spring will hold the screwed joint stably.

Compared with the traditionally rigid gimbals, the spring linker has quite small bending stiffness and of course still has the ability to transfer force and motion from one module of the robot to another one. It has couple of advantages illustrated as follows:

(a) No Singular Points. The traditional rigid universal joint can be applied in transmitting force and torque from one direction to another direction in 3D space. Nevertheless, there are usually some dead points on the boundary of its reachable space that constrains the range of the transmission angle. This singularity can be avoided in flexible spring linkages transmitting.

(b) Hollow Conformation. This nature helps the robots find their ways to pass through pneumatic lines and electric and signal cables.

(c) Length Shortened. The length can be eliminated to a minimum size due to their high flexibility and compact assembly.

(d) Crash Cushion. Pipes always have cracks, corruption pots, impurity, and something else in it. Therefore, there must be a lot of stochastic crashes that occurred to moving robot perform a great harm to the machine mechanism. The linker spring relaxes these strikes and makes the motion of robot more smooth.

(e) Energy Storage. The linker spring works as an energy storage unit if the action of telescopic mechanism could be properly controlled. The energy storage function improved the energy utilization ratio and synthesis characteristic of the robot.

With nominal diameter of $2 R$ and helix angle of $\theta$, the spring has the same circular cross sections from head to foot. $r$ is the radius of the spring wire whose original length is $l$. Before analysis, we assume the following. Firstly, the material is well distributed so that its elastic modulus and shear modulus remain constant everywhere [6]. Secondly, the deformation along the axis of spring wire is ignored for its tiny influence. Thirdly, the gravity is neglected and no contact happens. Lastly, in the deforming process, the rigid cross section keeps perpendicular to the axis line of spring wire all the time. All the assumptions made herein are reasonable, further meeting the hypothesis of Kirchhoff theory [40, 41]. Additionally, note that the compressive stiffness of a straight spring is equal to its tensile stiffness, so only the compressive stiffness will be referred to in the following description.

\section{Bending Stiffness}

3.1. Coordinate System Built. In order to describe the deformation, some reference frames have been built on the vertical posed spring. As shown in Figure 2, the general Descartes reference system $\mathrm{O}-\xi \eta \zeta$ is located at the start point $P_{0}$ at the bottom. Its $\zeta$-axis is set to be parallel with the central axis of the spring and the $\xi$-axis set horizontally left forward. An arc coordinate is built up from the start point $P_{0}$ so that every point along the axis of spring wire can be expressed as $P(s)$, where $s$ is the accumulated arc length. Translating $O-\xi \eta \zeta$ from start point $P_{0}$ to point $P$ yields local coordinate system $P-\xi \eta \zeta$. The rotated $P-\xi \eta \zeta$ around $\zeta$-axis by $\alpha$ is $P-\xi^{\prime} \eta^{\prime} \zeta$, which is then rotated around $\xi^{\prime}$-axis by the helix angle $\theta$ forming the principle coordinate system $P-x y z$ of the circular cross section. As the cross section rotates around the tangential direction to produce a new system $P-x^{\prime} y^{\prime} z^{\prime}$, the rotated angle is denoted as $\beta$. As a result, the three Euler angles $\alpha, \theta$, and $\beta$, which are all functions of the arc length $s$, together define the orientation of every cross section. Figure 3 shows the conversion process of different frames from beginning to end.

3.2. Initial State Description. Based on the three Euler angles, the curvature vector $\overrightarrow{w(s)}$ for a spatial curve has the following expression [42]:

$$
\left\{\begin{array}{l}
w_{1}(s)=\frac{\mathrm{d} \alpha(s)}{\mathrm{d} s} \sin \theta(s) \sin \beta(s)+\frac{\mathrm{d} \theta(s)}{\mathrm{d} s} \cos \beta(s), \\
w_{2}(s)=\frac{\mathrm{d} \alpha(s)}{\mathrm{d} s} \sin \theta(s) \cos \beta(s)-\frac{\mathrm{d} \theta(s)}{\mathrm{d} s} \sin \beta(s), \\
w_{3}(s)=\frac{\mathrm{d} \alpha(s)}{\mathrm{d} s} \sin \theta(s)+\frac{\mathrm{d} \beta(s)}{\mathrm{d} s}
\end{array}\right.
$$

For a spring shown in Figure 1 with circular cross section, the $\beta$ is zero [43]. Thus, the initial state of every piece of cross sections can be simplified to 


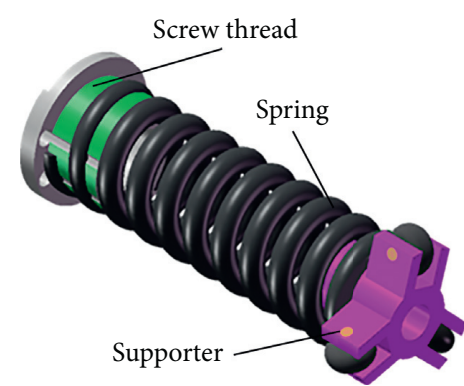

(a)

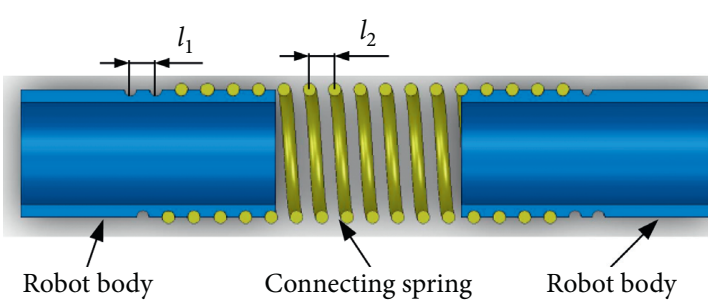

(b)

FIGURE 1: Spring linkage mechanism.

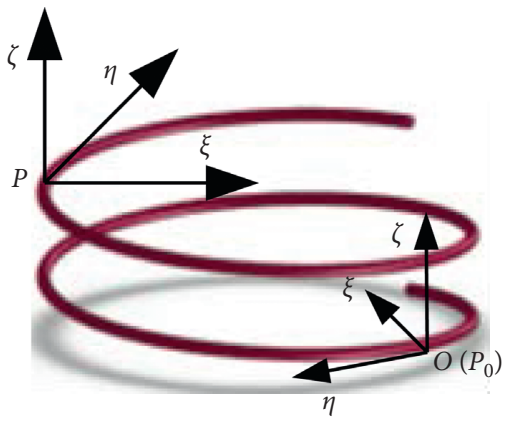

Figure 2: Coordinate systems.

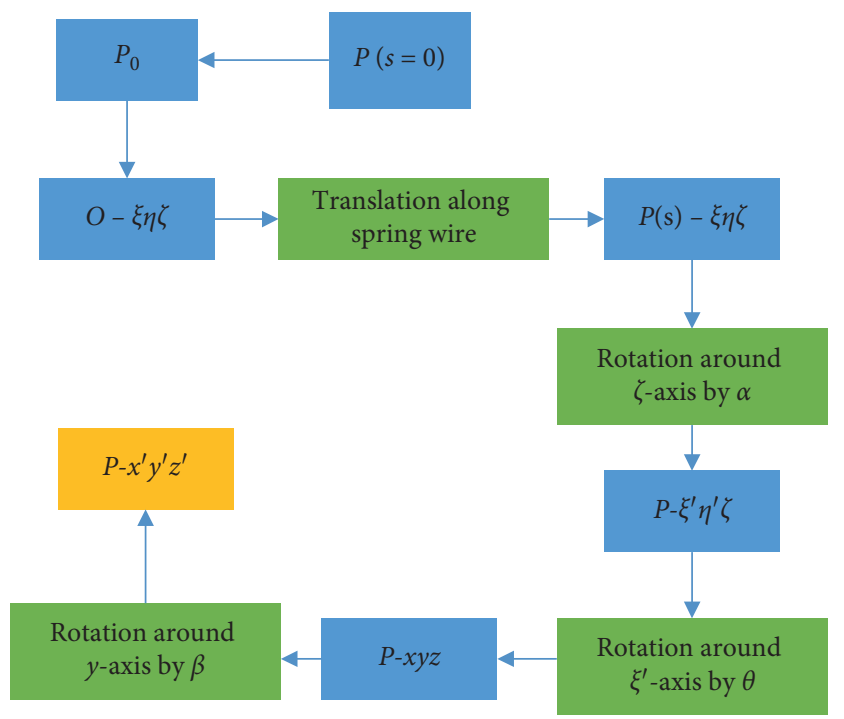

FIgURE 3: Conversion process of different frames.

$$
\left\{\begin{array}{l}
w_{1}^{0}=\frac{\mathrm{d} \theta_{0}}{\mathrm{~d} s}=\theta_{0}^{\prime}=0, \\
w_{2}^{0}=\frac{\mathrm{d} \alpha_{0}}{\mathrm{~d} s} \sin \theta_{0}=\alpha_{0}^{\prime} \sin \theta_{0}, \\
w_{3}^{0}=\frac{\mathrm{d} \alpha_{0}}{\mathrm{~d} s} \cos \theta_{0}+\frac{\mathrm{d} \beta_{0}}{\mathrm{~d} s}=\alpha_{0}^{\prime} \cos \theta_{0},
\end{array}\right.
$$

in which not only $\mathrm{d} \beta_{0} / \mathrm{d} s=0$, but also $\beta_{0}=0$. That is because the relative torsion angle and its derivative are both equal to zero as the spring stays free. Moreover, according to the parameters defined above, we have the relation shown as

$$
\frac{\mathrm{d} \alpha_{0}}{\mathrm{~d} s}=\frac{2 \pi}{\left((2 \pi R) /\left(\cos \theta_{0}\right)\right)}=\frac{\cos \theta_{0}}{R} .
$$

3.3. Applied Moment Description. Figure 4 shows the loading condition of the linkage spring as the in-pipe robot is moving inside elbows. Two ends of the connecting spring would be under the action of two equally opposite moments $M$, whose direction is along the $\xi$-axis of the general reference system $O-\xi \eta \zeta$.

In order to transfer the moment from the general coordinate system to the principle one, the relationship between these two is written down according to their definitions illustrated above:

$$
\left(\begin{array}{c}
\vec{I} \\
\vec{J} \\
\vec{K}
\end{array}\right)=\left(\begin{array}{ccc}
\cos \alpha & -\sin \alpha \cos \theta & \sin \alpha \sin \theta \\
\sin \alpha & \cos \theta \cos \alpha & -\cos \alpha \sin \theta \\
0 & \sin \theta & \cos \theta
\end{array}\right)\left(\begin{array}{c}
\vec{i} \\
\vec{j} \\
\vec{k}
\end{array}\right),
$$

in which, $(\vec{I} \vec{J} \vec{K})$ and $(\vec{i} \vec{j} \vec{k})$ are the coordinate basis vectors of general and principle reference systems, respectively [44]. Thus, the acting moment $M$ in $\xi$-axis direction can be transmitted to

$$
\begin{aligned}
M \vec{I} & =M_{1} \vec{i}+M_{2} \vec{j}+M_{3} \vec{k} \\
& =M(\cos \alpha \vec{i}-\sin \alpha \cos \theta \vec{j}+\sin \alpha \sin \theta \vec{k}) .
\end{aligned}
$$

3.4. Bent Spring Description. Under the action of moment $M$, the connecting spring gets bent around $\xi$-axis and two end faces turn to form an included angle $\gamma$. It is worth noticing that the helix angle $\theta$ cannot remain as a constant any more due to the flexural deformation of the spring. As a result, the curvature vector of the bent spring can be expressed under the principle coordinate system as

$$
\vec{w}=w_{1} \vec{i}+w_{2} \vec{j}+w_{3} \vec{k}=\theta^{\prime} \vec{i}+\gamma^{\prime} \vec{I}+\alpha^{\prime} \vec{K} .
$$

Substituting (4) into (6) yields 


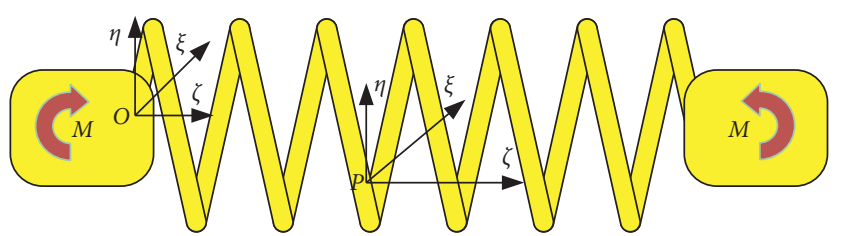

FIGURE 4: Spring under bending moment.

$$
\begin{aligned}
\vec{w} & =\left(\theta^{\prime}+\gamma^{\prime} \cos \alpha\right) \vec{i}+\left(\alpha^{\prime} \sin \theta-\gamma^{\prime} \cos \theta \sin \alpha\right) \vec{j} \\
& +\left(\alpha^{\prime} \cos \theta+\gamma^{\prime} \sin \theta \sin \alpha\right) \vec{k} .
\end{aligned}
$$

3.5. Solution. According to the Kirchhoff equation, for each piece of cross sections, we have

$$
\left\{\begin{array}{l}
M_{1}=E I_{x}\left(w_{1}-w_{1}^{0}\right) \\
M_{2}=E I_{y}\left(w_{2}-w_{2}^{0}\right) \\
M_{3}=G I_{z}\left(w_{3}-w_{3}^{0}\right)
\end{array}\right.
$$

in which, $E$ and $G$ are the elastic modulus and shear modulus of the spring material, respectively. $I_{x}$ and $I_{y}$ are two inertia moments of the related cross section around $x$ - and $y$-axis, while $I_{z}$ is its polar moment of inertia around $z$-axis. For the spring with circular cross sections, $2 I_{x}=2 I_{y}=I_{z}=\pi r^{4} / 2$.

By substituting (2), (5), and (7) into (8) simultaneously, the following holds:

$$
\left\{\begin{array}{l}
M \cos \alpha=E I_{x}\left(\theta^{\prime}+\gamma^{\prime} \cos \alpha\right)-M \sin \alpha \cos \theta \\
\quad=E I_{x}\left(\alpha^{\prime} \sin \theta-\gamma^{\prime} \cos \theta \sin \alpha-\alpha_{0}^{\prime} \sin \theta_{0}\right) \\
M \sin \alpha \sin \theta=2 G I_{x}\left(\alpha^{\prime} \cos \theta+\gamma^{\prime} \sin \theta \sin \alpha-\alpha_{0}^{\prime} \cos \theta_{0}\right)
\end{array}\right.
$$

By combining the last two equations in (9), the following relation holds:

$$
\begin{gathered}
\frac{M}{E I_{x}} \sin \alpha \cos \theta \cos \theta_{0}+\frac{M}{2 G I_{x}} \sin \alpha \sin \theta \sin \theta_{0} \\
=\alpha^{\prime} \sin \left(\theta_{0}-\theta\right)+\gamma^{\prime} \sin \alpha \cos \left(\theta-\theta_{0}\right) .
\end{gathered}
$$

Then, we solve (10) by recurring to (3) and taking $\theta=$ $\theta_{0}+\Delta \theta$ into consideration. The result is

$$
\begin{aligned}
\frac{M}{E I_{x}} & +\left(\frac{M}{2 G I_{x}}-\frac{M}{E I_{x}}\right)\left(\sin ^{2} \theta_{0}+\Delta \theta \sin \theta_{0} \cos \theta_{0}\right) \\
& =-\frac{\alpha^{\prime} \Delta \theta}{\sin \alpha}+\gamma^{\prime},
\end{aligned}
$$

in which, the second and higher order small quality are both neglected.

On the other hand, the substitution of $\theta=\theta_{0}+\Delta \theta$ into the first equation of (9) results in

$$
\mathrm{d} \Delta \theta=\left(\frac{M}{E I_{x}}-\gamma^{\prime}\right) \cos \alpha \mathrm{d} \alpha \frac{\mathrm{d} s}{\mathrm{~d} \alpha} .
$$

That is,

$$
-\frac{\alpha^{\prime}}{\sin \alpha} \Delta \theta=\gamma^{\prime}-\frac{M}{E I_{x}}
$$

Taking (13) back into (11) leads to

$2 \frac{M}{E I_{x}}+\left(\frac{M}{2 G I_{x}}-\frac{M}{E I_{x}}\right)\left(\sin ^{2} \theta_{0}+\Delta \theta \sin \theta_{0} \cos \theta_{0}\right)=2 \gamma^{\prime}$.

Multiplied by $\mathrm{d} s$ on the two sides, (14) takes integration along the whole spring wire:

$$
\begin{aligned}
& {\left[\frac{M}{E I_{x}}+\frac{1}{2}\left(\frac{M}{2 G I_{x}}-\frac{M}{E I_{x}}\right) \sin ^{2} \theta_{0}\right] l} \\
& \quad+\frac{1}{2}\left(\frac{M}{2 G I_{x}}-\frac{M}{E I_{x}}\right) \sin \theta_{0} \cos \theta_{0} \int_{l} \Delta \theta \mathrm{d} s \int_{l} \gamma^{\prime} \mathrm{d} s,
\end{aligned}
$$

in which, $l$ is the whole length of the spring coil. It can be expressed as

$$
l=\frac{2 n \pi R}{\cos \theta}
$$

Remember that the two ends of the spring are both fixed to the robot body, which means no variation or variation rate of the Euler angles has happened at those sections. Therefore, the integration of the second item laid on the left side of the equal sign in (15) equals to zero. Besides, the integration on the right hand is the sum of rotation of all the pieces of the cross sections, which is $\gamma$. Thus,

$$
\left[\frac{M}{E I_{x}}+\frac{1}{2}\left(\frac{M}{2 G I_{x}}-\frac{M}{E I_{x}}\right) \sin ^{2} \theta_{0}\right] l=\gamma .
$$

In this work, we define the bending stiffness $K_{b}$ of connecting spring as the ratio of the applied moment $M$ at its two ends to the resulting rotation angle $\gamma$. By taking $I_{x}=$ $\pi r^{4} / 4$ into consideration, the expression of result is

$$
K_{b}=\frac{M}{\gamma}=\frac{E G \pi r^{4}}{\left[4 G+(E-2 G) \sin ^{2} \theta_{0}\right] l} .
$$

By making use of (20) and (16), the bending stiffness of the connecting spring expressed in (18) can be rewritten down as

$$
K_{b}=\frac{E r^{4} \cos \theta_{0}}{4\left(2+\nu \sin ^{2} \theta_{0}\right) n R},
$$

in which the following relation was applied:

$$
\frac{E}{G}=2(1+\nu) \text {. }
$$

It can be seen from (18) that the bending stiffness $K_{b}$ is an absolutely nonlinear function, in terms of material and construction parameters. Meanwhile, it indicates that the 
bending stiffness $K_{b}$ of spring decreases with the raised initial helix angle or the elongated spring wire but increases as the radius of the spring wire grows thicker.

In particular, for $\theta_{0}=\pi / 2$, spring changes to straight rod with bending stiffness of

$$
\left.K_{b}\right|_{\theta_{0}=(\pi / 2)}=\frac{2 E I_{x}}{(2+v) l}
$$

The result shown in (21) is similar to but more accurate than the traditional one expressed as $\left.K_{b}\right|_{\theta_{0}=(\pi / 2)}=E I_{x} / l$.

It is worth remembering that the compressive stiffness of a spring has the definition [36]

$$
K=\frac{G r^{4}}{16 n R^{3}} .
$$

\section{Simulation and Verification}

It can be seen from (19) that the bending stiffness is a function of $n, R, r$, and $\theta$. In order to verify its rightness and find out its scope of application, this section makes some simulations that were performed using the following steps:

Step 1: select a set of parameters as an example to make model in SolidWorks

Step 2: import the model from SolidWorks into Workbench to get meshed and loaded

Step 3: make simulation on compressive stiffness and then compare the result with that calculated in (22) to ensure the validity of the simulation itself

Step 4: make simulation on bending stiffness and compare its result with that calculated in (19) to verify the correctness of (19)

Step 5: repeat operation from step 1 to step 4 with varying construction parameters $n, R, r$, and $\theta$, in order to find out its applicable scope

4.1. Data Selection. The parameter values used for the example simulation are listed in Table 1.

4.2. Simulation Performance. Based on the data listed in Table 1, a modified spring model was made as shown in Figure 5 which has two extra solid connectors for the sake of easy loading. Then, the model was imported from SolidWorks into Workbench for next simulation.

As verification of the simulation itself, the imported spring model was firstly simulated on compressive stiffness and then on bending stiffness. Figure 5 shows the result of spring under compressive force of $10 \mathrm{~N}$ that was acting along the spring axial. The result is $8.8581 \mathrm{~mm}$. Thus, the compressive stiffness of the spring is $1.13 \mathrm{~N} / \mathrm{mm}$, while the result of (22) is $1.20 \mathrm{~N} / \mathrm{mm}$. The similarity of the two results successfully ensured the validity of simulation.

Once the validity of simulation got verified by the comparison of calculation and simulation results, we began to make bending simulation. One of the results is shown in
TABLE 1: Spring structural parameter.

\begin{tabular}{lc}
\hline Item & Value \\
\hline Elastic modulus $(\mathrm{EPa})$ & $2 \times 10^{11}$ \\
Poisson ratio $v$ & 0.3 \\
Shear modulus $(\mathrm{GPa})$ & $0.77 \times 10^{11}$ \\
Radius of spring wire $r(\mathrm{~mm})$ & 0.5 \\
Normal radius of spring $R(\mathrm{~mm})$ & 5 \\
Active coils number $n$ & 8 \\
Pitch $l_{2}(\mathrm{~mm})$ & 10 \\
Spring length $L(\mathrm{~mm})$ & 80 \\
Length of spring wire $l(\mathrm{~mm})$ & 263.753 \\
Helix angle $\theta_{0}(\mathrm{deg})$ & 17.66 \\
\hline
\end{tabular}

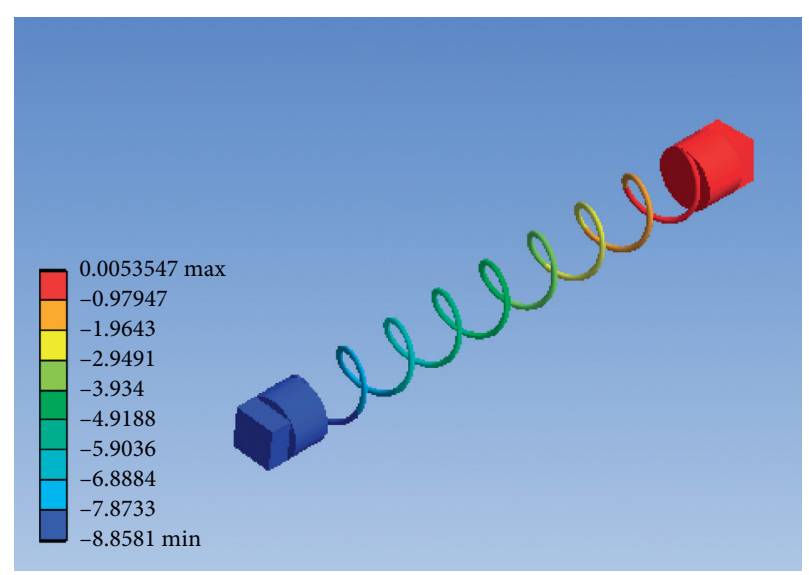

FIGURE 5: Spring deformation under compression.

Figure 6, with two moments of $10 \mathrm{Nmm}$ acting in the way shown in Figure 4. In order to find out the rotated angle of the end face, we made use of the maximum and minimum displacement in the axial direction. They are $1.8787 \mathrm{~mm}$ and $-1.879 \mathrm{~mm}$, respectively, in Figure 6.

By considering the geometry relationship shown in Figure 7 , the following equation holds:

$$
\frac{\min }{h_{1}}=\frac{\max }{h_{2}}=\tan \gamma,
$$

in which, $h_{1}+h_{2}$ is the spring outside diameter, equal to $2(R+r)$. As a result, the rotated angle $\gamma$ is 0.329 , and then the bending rigidity is $30.379 \mathrm{Nmm} / \mathrm{rad}$. On the other hand, the corresponding result can be obtained by substituting all needed values shown in Table 1 into (19). It is $31.2 \mathrm{Nmm} / \mathrm{rad}$, very close to that of simulation result.

\section{Results and Discussion}

After a demonstrative simulation, the parameters that appeared in (19) were all changed to reconstruct the spring and redo the simulation, one at a time. Firstly, the active coils number $n$ was chosen to be changed, from 4 to 18 . Figure 8 shows the results of both simulation result represented by star and the calculation result represented by line.

It can be seen from Figure 8 that both the compressive and bending rigidities have their calculated and simulated 


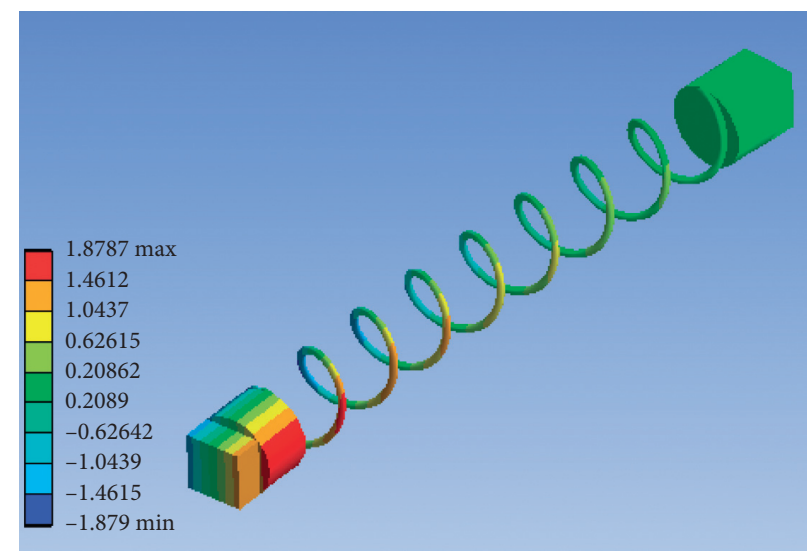

Figure 6: Spring deformation under bending moment.

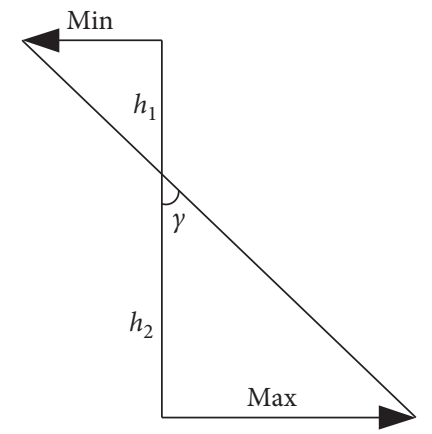

FIGURE 7: Geometry relation between $\gamma$ and two maximum deformation values.

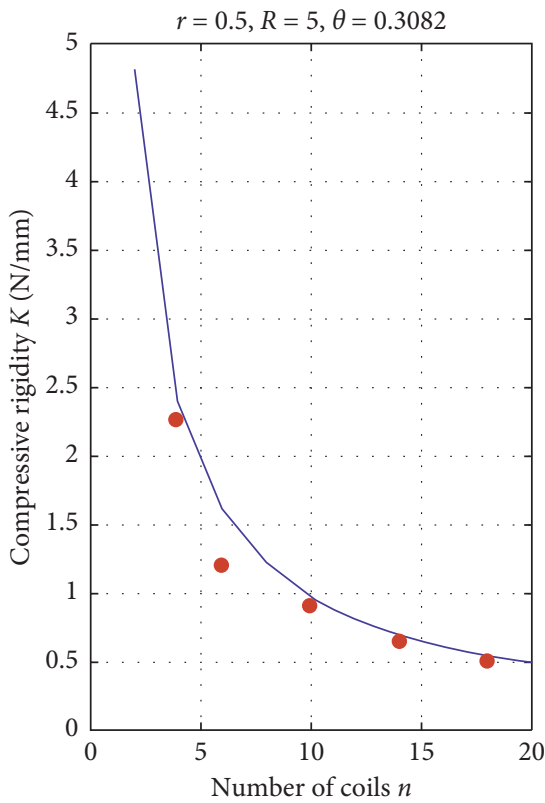

(a)

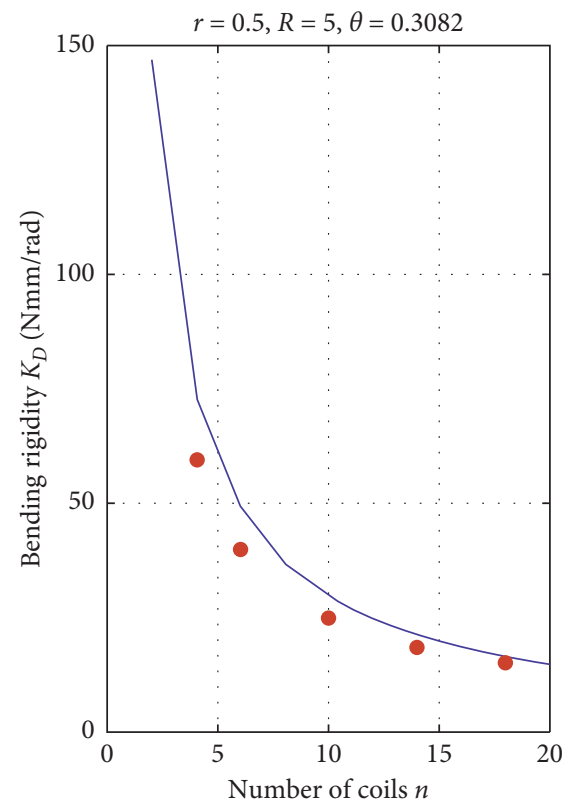

(b)

Figure 8: Influence of active coils number $n$ (一: calculated; ${ }^{*}$ : simulated).

results staying close to each other after $n$ grows larger than 4 . The active coils number $n$ provides an inverse proportion effect on two rigidities.
The nominal radius $R$ was the second one to be changed from $6 \mathrm{~mm}$ to $12 \mathrm{~mm}$. The results shown in Figure 9 also indicate an inverse effect of normal radius $R$ on rigidities. 


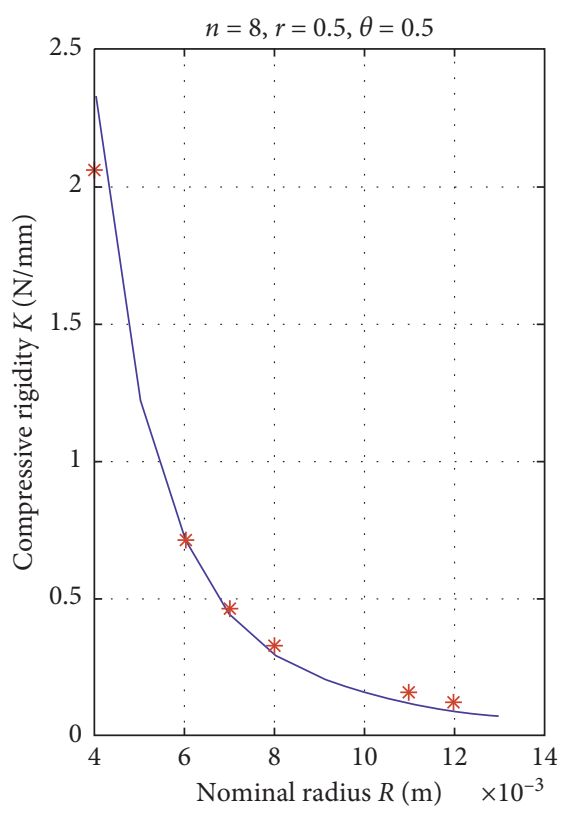

(a)

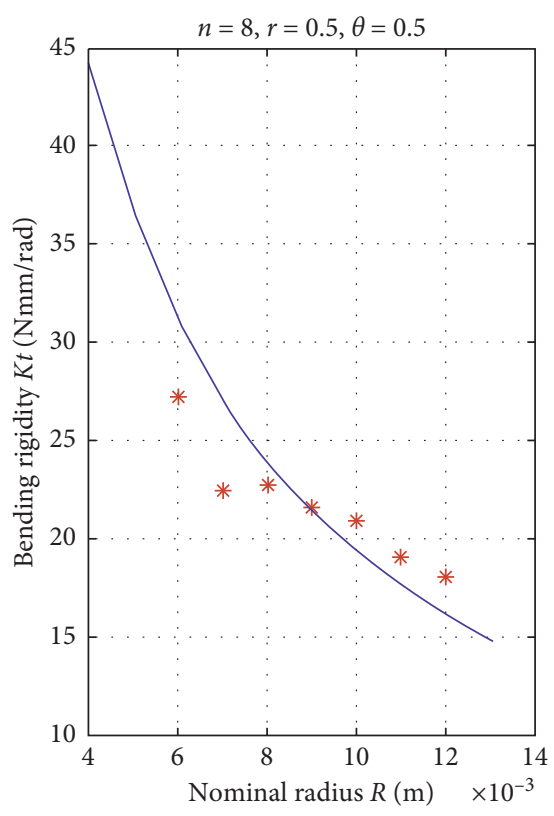

(b)

FIGURE 9: Influence of nominal radius $R$ (一: calculated; *: simulated).

The effect of coil radius is positive, according to the results of Figure 10. In particular, the square symbol " $\square$ " describes the results of Figures 5 and 6.

Notice that there is no helix angle $\theta_{0}$ involved in the expression of compressive rigidity shown in (22). This is a big problem. However, the simulation result of spring bending rigidity indeed changes with varying helix angles as shown in Figure 11(a), totally different from the horizontal line representing the calculation result. Figure 11(b) shows that the calculation and simulation results meet at the helix angle of $7^{\circ}$, although diverged quickly before and after. Therefore, we can obtain a conclusion here that the expression used for calculation spring bending rigidity is of the best utility when helix angle $\theta_{0}$ is set around 7 .

\section{Compression and Bending Stiffness Balancing}

All simulations made above have verified the correctness of the deduced bending expression shown in (19). In order to find out the relationship between $K$ and $K_{b}$, we substitute (22) into (18), and the result is

$$
K_{b}=K \frac{8(1+\nu) \cos \theta_{0} R^{2}}{2+\nu \sin ^{2} \theta_{0}} .
$$

It seems that to modify the helix angle $\theta_{0}$ may be an effective way to change $K_{b}$ while leaving $K$ unchanged, because there is no helix angle $\theta_{0}$ involved in $K$. However, the results shown in Figure 11 denied the possibility of this alternation.
Besides the material parameter $v$, there is only the nominal radius $R$ left. Therefore, to modify $R$ is an effective way to adjust $K_{b}$ while keeping the $K$ unchanged.

All the notations mentioned above are listed in Table 2, as well as their meanings.

\section{Experiments}

For in-pipe robots, those turning a corner, an elastic connector is always required [45]. In order to achieve effective power transmission from one module to another, the spring compression stiffness should be large enough. Meanwhile, the bending elastic connector must produce normal pressure against the inner pipe wall when passing through a corner. The normal pressure will generate friction that hinders the forward movement of in-pipe robot. The above calculation and simulation results tell us that the balance between compression stiffness and bending stiffness could be achieved, according to (24).

In this part, we applied some springs with different shape parameters to assemble the same robot. Then we make velocity test when passing through a bending pipe. The maximum velocity will express the best combination of spring shape parameters and also the best balance between compression and bending stiffness. Figure 12 shows the basic components of an in-pipe robot whose elastic connector can move smoothly through the bent pipe thanks to the spring. Figure 13 shows the experiment platform for the velocity test. It has in-pipe robot drive circuit and control algorithm for driving, as well as bent pipe for running environment. 


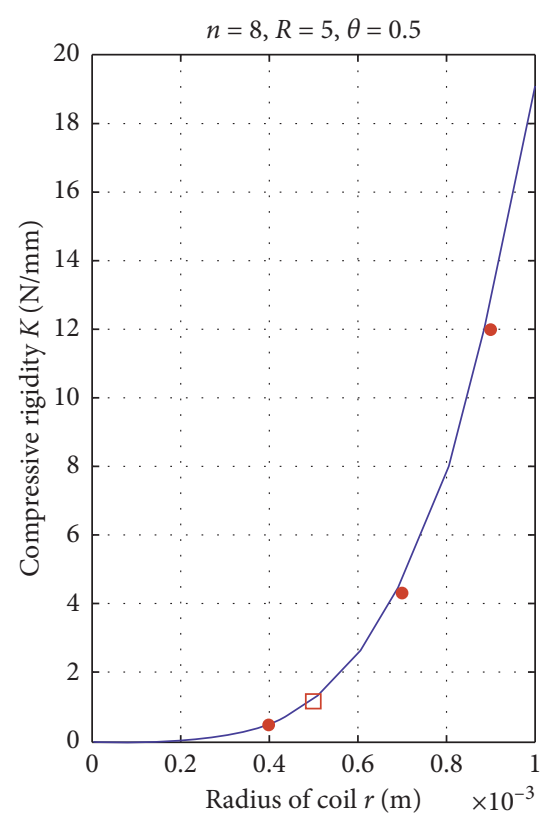

(a)

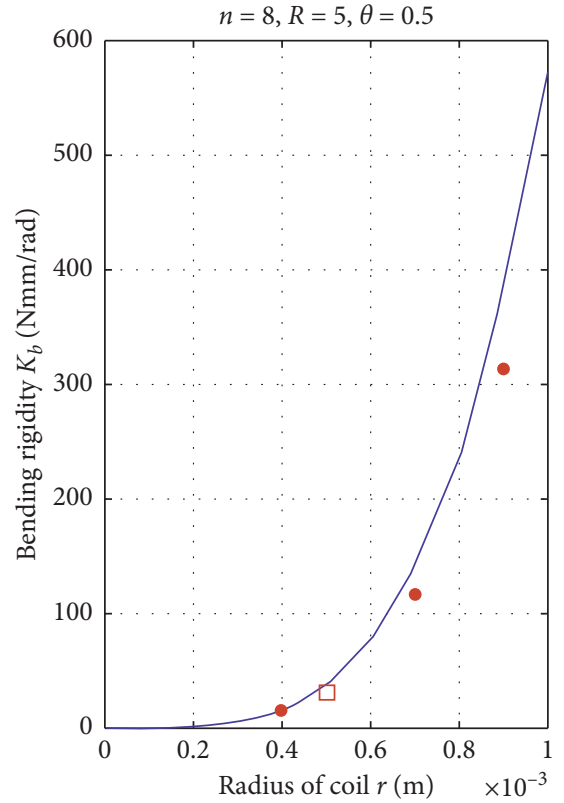

(b)

FIGURE 10: Influence of coil radius $r$ (一: calculated; ${ }^{*}$ : simulated).

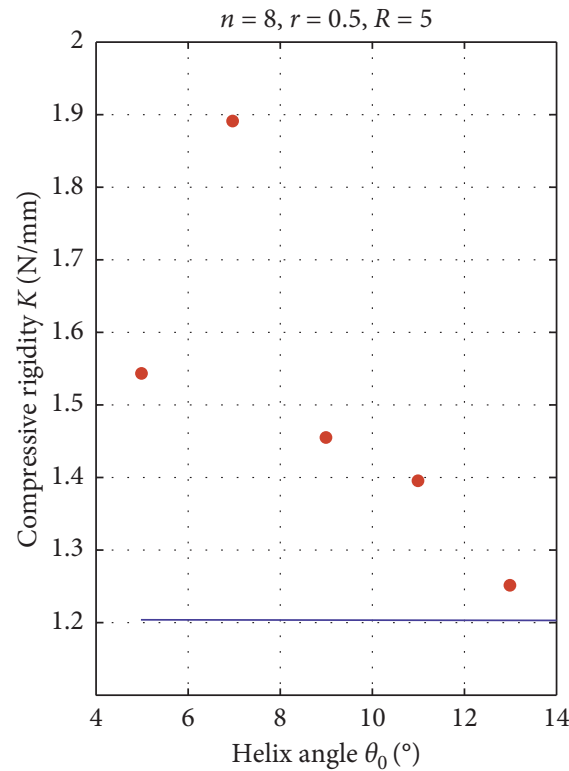

(a)

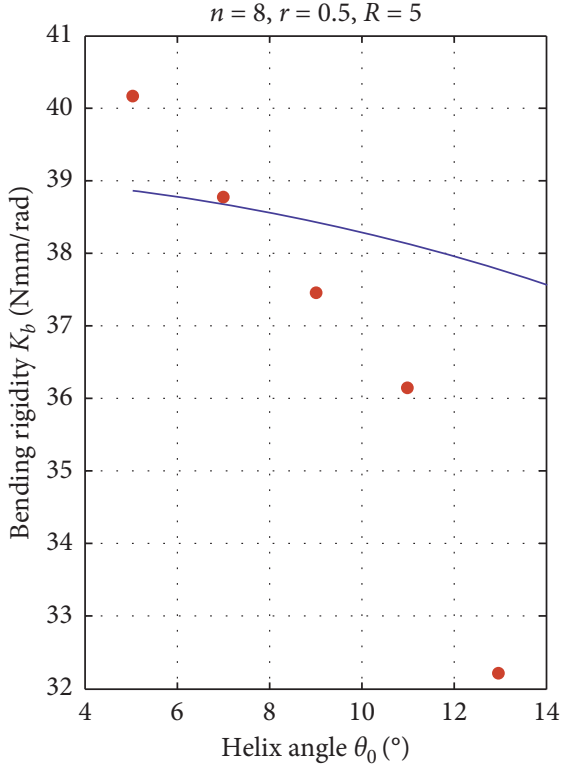

(b)

Figure 11: Influence of helix angle $\theta_{0}$ (一: calculated; *: simulated).

During the experiment process, we kept spring material and normal radius constant, $R=10 \mathrm{~mm}$, and changed the wire radius, helix angle, and active coil number one by one.

It can be seen from the experiment results that the spring compression and bending stiffness both increase dramatically with bigger coil radius. The maximum speed comes at the $6^{\text {th }}$ parameter set in Table 3 . The bending passing velocity increased initially with bigger $K$; however, it got slower with lasting increasing $K$. It indicates the same trends with calculation and simulation result. 
TABLE 2: Character definition table.

\begin{tabular}{|c|c|}
\hline Notation & Meaning \\
\hline$E$ & Material elastic modulus \\
\hline G & Material shear modulus \\
\hline$H_{1}, h_{2}$ & $\begin{array}{c}\text { The only two components of the outer diameter, corresponding to the mix and max deformation in each } \\
\text { cross section }\end{array}$ \\
\hline$\left(\begin{array}{lll}\vec{i} & \vec{j} & \vec{k}\end{array}\right)$ & Coordinate basis vectors of principle reference systems \\
\hline$(\vec{I} \vec{J} \vec{K})$ & Coordinate basis vectors of general reference systems \\
\hline$I_{x}, I_{y}, I_{z}$ & Inertia moment around $\mathrm{x}^{-}, \mathrm{y}-$, and $\mathrm{z}$-axis \\
\hline$K$ & Spring compression stiffness \\
\hline$K_{b}$ & Spring bending stiffness \\
\hline$L$ & Spring wire length \\
\hline$l_{1}$ & Screw pitch of robot module \\
\hline$l_{2}$ & Screw pitch of spring \\
\hline$M$ & Acting moment \\
\hline$O-\xi \eta \zeta$ & General Descartes reference system \\
\hline$P_{0}$ & Start point at bottom of spring \\
\hline$P(s)$ & Point along the axis of spring wire \\
\hline$P-x y z$ & Principle coordinate system \\
\hline$P-\xi \eta \zeta$ & Local coordinate system \\
\hline$R$ & Spring wire radius \\
\hline$R$ & Spring nominal radius \\
\hline$S$ & Accumulated arc length \\
\hline $\overrightarrow{w(s)}=\left(w_{1}(s), w_{2}(s), w_{3}(s)\right)$ & Curvature vector \\
\hline A & Rotation angle about $\zeta$-axis \\
\hline B & Rotation angle of the cross section around the tangential direction \\
\hline$\Gamma$ & Included angle \\
\hline$v$ & Poisson ratio \\
\hline$\Theta$ & Helix angle \\
\hline$\theta_{0}$ & Initial value of $\theta$ \\
\hline
\end{tabular}

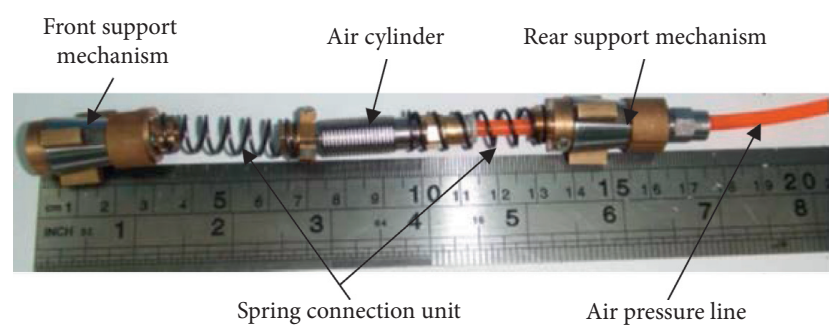

FIgURE 12: In-pipe robot with elastic connector.

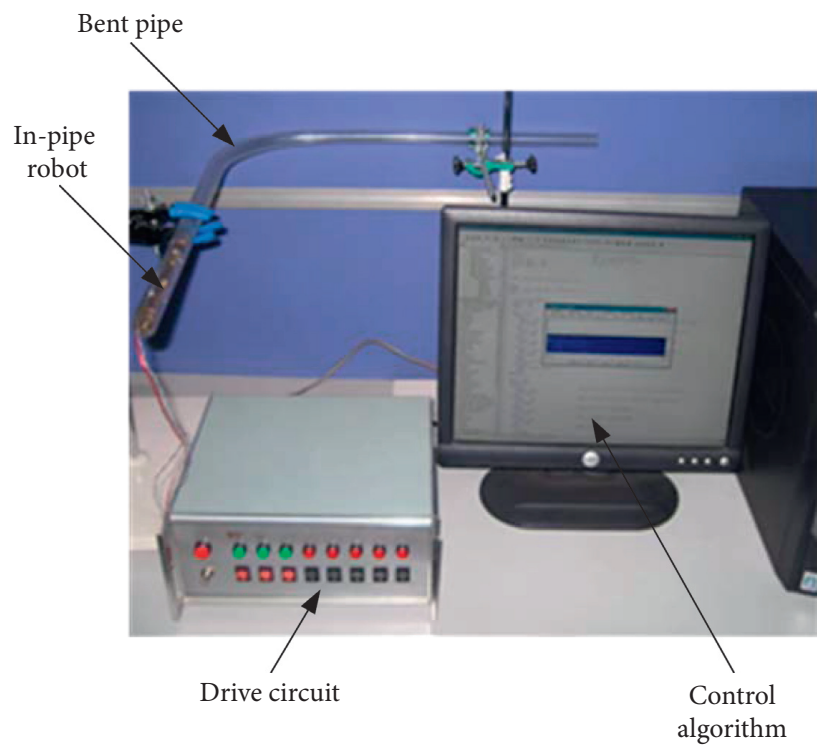

FIGURE 13: Experiment equipment for the robot velocity test. 
TABLE 3: Experiment results.

\begin{tabular}{|c|c|c|c|c|c|c|}
\hline No. & Coil radius $r(\mathrm{~mm})$ & $n$ & Helix angle $\theta_{0}(\mathrm{rad})$ & Compression stiffness $K(\mathrm{~N} / \mathrm{m})$ & Bending stiffness $K_{b}(\mathrm{~N} / \mathrm{m})$ & Velocity $(\mathrm{mm} / \mathrm{s})$ \\
\hline 1 & 0.5 & 6 & 0.1 & 201 & 259 & 3.3323 \\
\hline 2 & 0.5 & 6 & 0.2 & 201 & 254 & 4.1148 \\
\hline 3 & 0.5 & 6 & 0.3 & 201 & 246 & 4.7221 \\
\hline 4 & 1 & 10 & 0.1 & 1925 & 2484 & 4.7324 \\
\hline 5 & 1 & 10 & 0.2 & 1925 & 2436 & 5.2970 \\
\hline 6 & 1 & 10 & 0.3 & 1925 & 2357 & 5.6852 \\
\hline 7 & 1.5 & 14 & 0.1 & 6961 & 8982 & 2.6500 \\
\hline 8 & 1.5 & 14 & 0.2 & 6961 & 8808 & 3.1622 \\
\hline 9 & 1.5 & 14 & 0.3 & 6961 & 8525 & 3.6225 \\
\hline
\end{tabular}

\section{Conclusions}

In order to improve the characteristic of connecting spring applied to in-pipe robot, a balance is obtained between the compressive stiffness and the bending stiffness of the spring:

(1) The detail of the spring linkage mechanism was introduced at first. It was simple, reliable, and really suitable for the application in in-pipe robot.

(2) In order to get a higher bending passing speed, the spring linkage should be neither too soft nor too hard. The compression and bending rigidity need an optimization design.

(3) The expression for spring bending rigidity calculation was deduced. Combining the simulation and experiment results, we found the suitable method to optimize the spring. The most sensitive parameter was the helix angle $\theta_{0}$.

\section{Data Availability}

The data used to support the findings of this study are available from the corresponding author upon request.

\section{Conflicts of Interest}

The authors declare that they have no conflicts of interest regarding the publication of this paper.

\section{Acknowledgments}

This work was supported by the Key Research and Development Plan of Shandong Province (grant no. 2019GGX104044) and the Major Scientific and Technological Innovation Projects of Shandong Province (grant no. 2019JZZY020323).

\section{References}

[1] Y. S. Zhang, S. Y. Jiang, X. W. Zhang, X. Y. Ruan, and D. M. Guo, "A variable-diameter capsule robot based on multiple wedge effects," IEEE/ASME Transactions on Mechatronics, vol. 16, no. 2, pp. 241-254, 2011.

[2] M. Ono and S. Kato, "A study of an earthworm type inspection robot movable in long pipes," International Journal of Advanced Robotic Systems, vol. 7, no. 1, pp. 85-90, 2010.
[3] M. M. Moghaddam, M. Arbabtafti, and A. Hadi, "In-pipe inspection crawler adaptable to the pipe interior diameter," International Journal of Robotics \& Automation, vol. 26, no. 2, pp. 135-145, 2011.

[4] J. Chen, T. Chen, and Z. Q. Deng, "Dynamic properties on double driving articulated in-pipe robot inspecting system," Applied Mechanics and Materials, vol. 128-129, pp. 14301433, 2012.

[5] Z. Hu, Y. Wang, S. Yu, and G. Cui, "A method to enhance fidelity of force feedback control in virtual and human-robot micro interaction cardiovascular intervention surgery," The International Journal of Advanced Manufacturing Technology, vol. 105, no. 12, pp. 4883-4897, 2019.

[6] H. Tourajizadeh, M. Rezaei, and A. H. Sedigh, "Optimal control of screw in-pipe inspection robot with controllable pitch rate," Journal of Intelligent \& Robotic Systems, vol. 90, no. 3-4, pp. 269-286, 2018.

[7] T. Sudhawiyangkul, K. Yoshida, S. I. Eom, and J.-w. Kim, “A novel bending microactuator with integrated flexible electrorheological microvalves using an alternating pressure source for multi-actuator systems," Microsystem Technologies, vol. 26, no. 5, pp. 1507-1519, 2020.

[8] Q. Liu, J. Zhao, H. Zhu, W. Zheng, and Y. Yang, "A novel double bevel support structure for downhole robot," Arabian Journal for Science and Engineering, vol. 44, no. 2, pp. 10691079, 2019.

[9] S. Yaqub, A. Ali, M. Usman et al., "A spiral curve gait design for a modular snake robot moving on a pipe," International Journal of Control, Automation and Systems, vol. 17, no. 10, pp. 2565-2573, 2019.

[10] Y. Shi, Z. Mu, M. Cai, H. Song, and Y. Wang, "Advances in motion control of gas pipeline detection robot," Science China Technological Sciences, vol. 63, no. 5, pp. 877-878, 2020.

[11] F. Moreira, A. Abundis, M. Aguirre, J. Castillo, and P. A. Bhounsule, "An inchworm-inspired robot based on modular body, electronics and passive friction pads performing the two-anchor crawl gait," Journal of Bionic Engineering, vol. 15, no. 5, pp. 820-826, 2018.

[12] Z. Wang, S. Guo, Q. Fu, and J. Guo, "Characteristic evaluation of a magnetic-actuated microrobot in pipe with screw jet motion," Microsystem Technologies, vol. 25, no. 2, pp. 719-727, 2019.

[13] H. Schempf, E. Mutschler, A. Gavaert, G. Skoptsov, and W. Crowley, "Visual and nondestructive evaluation inspection of live gas mains using the explorer (TM) family of pipe robots," Journal of Field Robotics, vol. 27, no. 3, pp. 217-249, 2010.

[14] O. Adria, H. Streich, and J. Hertzberg, "Dynamic replanning in uncertain environments for a sewer inspection robot," International Journal of Advanced Robotic Systems, vol. 1, no. 1, pp. 33-38, 2008. 
[15] S. Wakimoto, J. Nakajima, M. Takata, T. Kanda, and K. Suzumori, A Micro Snake-like Robot for Small Pipe Inspection, Institute of Electrical and Electronics Engineers Inc, Nagoya, Japan, 2003.

[16] S. Hirose, H. Ohno, T. Mitsui, and K. Suyama, "Design of inpipe inspection vehicles for 25,50,150 pipes," in Proceedings of the IEEE International Conference on Robotics and Automation, pp. 2309-2314, Detroit, MI, USA, May 1999.

[17] C. Lin, Y. Hu, Y. Wei, and Z. Cai, "Characteristics analysis of supporting and locking mechanism based on the non-circular gear compound transmission," Journal Of Mechanical Science And Technology, vol. 34, no. 6, pp. 2561-2571, 2020.

[18] H. Takeshima and T. Takayama, "Development of a steerable in-pipe locomotive device with six braided tubes," ROBOMECH Journal, vol. 5, no. 1, 2018.

[19] M. Muramatsu, N. Namiki, R. Koyama, and Y. Suga, Autonomous Mobile Robot in Pipe for Piping Operations, Institute of Electrical and Electronics Engineers Inc, Takamatsu, Japan, 2000.

[20] S. Iwashina, I. Hayashi, N. Iwatsuki, and K. Nakamura, Development of In-Pipe Operation Micro Robots, IEEE, Nagoya, Japan, 1994.

[21] C. Q. Xu, Research on Design and Motion Stability of a Novel Creeping Micro In-Pipe Robot, National University of Defense Technology, Changsha, China, 2010.

[22] I. Hayashi, N. Iwatsuki, and S. Iwashina, Running Characteristics of a Screw-Principle Microrobot in a Small Bent Pipe, IEEE, Nagoya, Japan, 1995.

[23] L. Brown, J. Carrasco, S. Watson, and B. Lennox, "Elbow detection in pipes for autonomous navigation of inspection robots," Journal of Intelligent \& Robotic Systems, vol. 95, no. 2, pp. 527-541, 2019.

[24] Q. Tu, Q. Liu, T. Ren, and Y. Li, "Obstacle crossing and traction performance of active and passive screw pipeline robots," Journal of Mechanical Science and Technology, vol. 33, no. 5, pp. 2417-2427, 2019.

[25] Q. Liu, J. Zhao, H. Zhu, and W. Zhang, "Mechanical model of drilling robot driven by the differential pressure of drilling fluid," Arabian Journal for Science and Engineering, vol. 44, no. 2, pp. 1447-1458, 2019.

[26] S. Perreault, P. Cardou, and C. Gosselin, "Approximate static balancing of a planar parallel cable-driven mechanism based on four-bar linkages and springs," Mechanism and Machine Theory, vol. 79, pp. 64-79, 2014.

[27] F. B. Liu and Y. M. Cheng, "The improved element-free galerkin method based on the nonsingular weight functions for inhomogeneous swelling of polymer gels," International Journal of Applied Mechanics, vol. 10, Article ID 18500474, 2018.

[28] J. Cheng, "Data analysis of the factors influencing the industrial land leasing in Shanghai based on mathematical models," Mathematical Problems in Engineering, vol. 2020, Article ID 9346863, 11 pages, 2020.

[29] L. Chen, H. P. Ma, and Y. M. Cheng, "Combining the complex variable reproducing kernel particle method and the finite element method for solving transient heat conduction problems," Chinese Physics, vol. 22, no. 5, Article ID 050202, 2013.

[30] M. Peng and Y. Cheng, "A boundary element-free method (BEFM) for two-dimensional potential problems," Engineering Analysis with Boundary Elements, vol. 33, no. 1, pp. 77-82, 2009.

[31] K. M. Liew and Y. Cheng, "Complex variable boundary element-free method for two-dimensional elastodynamic problems," Computer Methods in Applied Mechanics and Engineering, vol. 198, no. 49-52, pp. 3925-3933, 2009.

[32] Z. Liu, G. Wei, and Z. Wang, "The radial basis reproducing kernel particle method for geometrically nonlinear problem of functionally graded materials," Applied Mathematical Modelling, vol. 85, pp. 244-272, 2020.

[33] Z. Liu, H. F. Gao, G. F. Wei, and Z. M. Wang, "The meshfree analysis of elasticity problem utilizing radial basisreproducing kernel particle method," Results in Physics, vol. 17, Article ID 103037, 2020.

[34] Z. Liu, G. Wei, and Z. Wang, "Numerical solution of functionally graded materials based on radial basis reproducing kernel particle method," Engineering Analysis with Boundary Elements, vol. 111, pp. 32-43, 2020.

[35] Z. Liu, G. F. Wei, and Z. M. Wang, "Numerical analysis of functionally graded materials using reproducing kernel particle method," International Journal of Applied Mechanics, vol. 11, no. 6, Article ID 1950060, 2019.

[36] B. H. Wang, Y. Q. Ma, and Y. M. Cheng, "The improved complex variable element-free Galerkin method for bending problem of thin plate on elastic foundations," International Journal of Applied Mechanics, vol. 11, no. 10, Article ID 1950105, 2019.

[37] Q. Wu, F. B. Liu, and Y. M. Cheng, "The interpolating element-free Galerkin method for three-dimensional elastoplasticity problems," Engineering Analysis with Boundary Elements, vol. 115, pp. 156-167, 2020.

[38] F. B. Liu, Q. Wu, and Y. M. Cheng, "A meshless method based on the nonsingular weight functions for elastoplastic large deformation problems," International Journal of Applied Mechanics, vol. 11, no. 1, Article ID 1950006, 2019.

[39] P. P. Peng, Q. Wu, and Y. M. Cheng, "The dimension splitting reproducing kernel particle method for three-dimensional potential problems," International Journal for Numerical Methods in Engineering, vol. 121, no. 1, pp. 146-164, 2020.

[40] A. E. H. Love, A Treatise on the Mathematical Theory of Elasticity, Dover Publications, New York, NY, USA, 1927.

[41] N. Eqra, S. Taghvaei, and R. Vatankhah, "Optimal kinematic design of a single-DOF planar grasper based on metaheuristic optimization," Journal of the Brazilian Society of Mechanical Sciences and Engineering, vol. 41, no. 10, 2019.

[42] Y. Z. Liu, Nonlinear Mechanics of Thin Elastic Rod-Theoretical Basis of Mechanical Model of DNA, Qtinghua University Press, Beijing, China, 2006.

[43] Y. Miyazaki and K. Kondo, "Analytical solution of spatial elastica and its application to kinking problem," International Journal of Solids and Structures, vol. 34, no. 27, pp. 3619-3636, 1997.

[44] R. Huang, S. J. Zheng, Z. S. Liu, and T. Y. Ng, "Recent advances of the constitutive models of smart materials-hydrogels and shape memory polymers," International Journal of Applied Mechanics, vol. 12, no. 2, Article ID 2050014, 2020.

[45] B. Arun Sundaram, K. Kesavan, and S. Parivallal, "Recent advances in health monitoring and assessment of in-service oil and gas buried pipelines," Journal of The Institution of Engineers (India): Series A, vol. 99, no. 4, pp. 729-740, 2018. 